\title{
LA POLÍTICA DE LA FRONTERA. ENTREVISTA A SANDRO MEZZADRA Y BRETT NEILSON
}

\section{Mary Luz Estupiñán Serrano ${ }^{1}$}

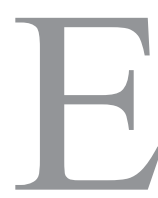

N Diciembre de 2016 la editorial Tinta Limón publicó en BueNos Aires La frontera como método o la multiplicación del trabajo, de Sandro Mezzadra y Brett Neilson, cuya primera versión salió en inglés en 2013. En esta oportunidad conversamos con los autores sobre las implicancias teórico-conceptuales de su propuesta. Abordamos la polisemia de la noción de frontera que ellos colocan en términos de proliferación; la necesidad de desbordar la centralidad de los muros como ícono por excelencia de la frontera geopolítica del presente; la redefinición de los conceptos claves del pensamiento político moderno operada por la globalización; la multiplicación del trabajo; así como los desafíos que plantean las luchas migratorias no solo en el plano político, sino también para la redefinición de lo común.

Sandro Mezzadra es profesor de filosofía política en la Universidad de Bolonia (Italia). Ha publicado trabajos sobre migraciones, capitalismo contemporáneo y globalización. Brett Nielson es profesor de teoría social y cultural en la Western Sydney University (Australia). Sus líneas de trabajo se centran en la precariedad laboral, la globalización y el capitalismo contemporáneo. En la actualidad los dos participan en un proyecto denominado Logistical Worlds. Infrastructure, Software, Labour.

Hasta hace muy poco la idea de frontera estaba asociada a un puñado de definiciones muy precisas que podrían resumirse en disciplinares, geográficas y simbólicas. Sin embargo, esta triada de asociaciones se ha visto desbordada dando

1 Doctora en Estudios Latinoamericanos por la Universidad de Chile. Investigadora postdoctoral FONDECYT, adscrita a la Universidad Metropolitana de Ciencias de la Educación (UMCE, Chile). La presente entrevista se enmarca dentro del proyecto $\mathrm{n}^{\circ} 3150589$. 


\section{lugar a lo que ustedes denominan una "proliferación de fronteras", que va desde fronteras espaciales, hasta lingüísticas pasando por fronteras sociales, económi- cas, temporales y jurídicas. Ante dicha polisemia ustedes proponen adoptar la frontera como método ¿En qué consiste este abordaje?}

En cierto modo, estamos convencidos de que una de las características fundamentales de lo que suele referirse como globalización radica precisamente en el hecho de que las fronteras [borders $]^{2}$ están cada vez más sometidas a presión. Sin embargo, esto no se corresponde en absoluto con una disminución de su relevancia y para entenderlo no era necesario esperar la suerte de reacción nacionalista que estamos experimentando en la actualidad en muchas partes del mundo. La noción de "apartheid global", que subyace a la relevancia de dispositivos fronterizos específicos, empezó a circular entre los debates críticos a principios de los años noventa, al tiempo que Étienne Balibar -quien ha jugado un papel importante en hacer de la frontera [border] un asunto central de la teoría política-comenzó a hablar, por esos mismos años, de un "apartheid europeo". ${ }^{3}$ Nuestro propio interés en el tema de la frontera emergió de las prácticas activistas relacionadas con la migración. Y de nuevo: desde inicio de los 90, tanto en Europa como en Australia, era factible ver que las fronteras (algunas de ellas mucho más que otras, por supuesto) estaban emergiendo como lugares políticos fundamentales, donde las prácticas de cruce fronterizo se estaban intersectando con las prácticas de reforzamiento de las fronteras con consecuencias a menudo letales. Y es precisamente siguiendo la pista de los migrantes que hemos podido concluir que esta nueva prominencia de las fronteras corre paralela a los procesos de flexibilización y desplazamiento de las fronteras mismas. Por una parte, los migrantes enfrentan muy a menudo la frontera mucho antes de la demarcación geopolítica lineal que intentan cruzar (en los estudios críticos de frontera este proceso recibe con frecuencia el nombre de "externalización"). Por otra parte, la sombra de la frontera sigue a los migrantes más allá del momento preciso del cruce de esa línea demarcada, modela sus biografías, emerge de formas heterogéneas (como

2 Border suele ser traducido en español como frontera, pero también refiere literalmente borde, límite. Sin embargo, hay otras palabras en inglés que tienen significados similares como boundary y frontier. Los autores usan las tres para indicar la polisemia del tema a tratar, de modo que traduciremos border como frontera, boundary como linde o línea y frontier como confín. De todas maneras, queremos llamar la atención sobre las otras posibilidades de traducción del primer término para mejor aprehender la complejidad de la que se intenta dar cuenta. Como la primera es la de mayor uso, indicaremos entre paréntesis el uso de la dos últimas.

3 BALIBAR, E, Les Frontières de la Democratie. Paris: La Découverte, 1992. 
límites [boundaries] urbanos, lingüísticos, étnicos) al interior del mismo espacio que las fronteras geopolíticas deberían supuestamente circunscribir. Tenemos la impresión que desde este punto de vista hay una diferencia esencial con respecto a las anteriores experiencias de migración. La crisis de los patrones de integración, la precarización del estatus de los migrantes tanto en términos legales como en lo que respecta a su posición en el mercado laboral, abren espacios dentro de los cuales las operaciones de una amplia y heterogénea gama de dispositivos fronterizos son mucho más invasivas que antes. Partiendo de esta impresión, comenzamos a investigar las fronteras tanto desde el ángulo de la proliferación de su existencia como desde el punto de vista de lo que llamamos su "heterogeneización". Estamos convencidos de que estos procesos son claves en la reorganización contemporánea del poder, de la dominación y de la explotación y que las luchas a su alrededor -que involucran a los migrantes, pero también a otros sujetos-, tienen papeles centrales a desempeñar en una nueva política de liberación.

En un sentido un tanto diferente, es importante destacar que el campo semántico de la frontera siempre ha sido profundamente heterogéneo. La mayoría de sus componentes (desde lo simbólico a lo cognitivo, desde lo lingüístico a lo económico) se mantuvieron articulados en la modernidad, para decirlo de manera resumida, por la metáfora del límite [boundary] lineal, que funcionaba como una especie de línea "magnética". La historia de este límite lineal, su origen en Europa y su disputada y desigual proyección global a través del colonialismo y el imperialismo, está lejos de ser sencilla -e intentamos reconstruirla en el libro. Pero lo que quizás sea más importante señalar aquí es que actualmente estamos viviendo un proceso de desarticulación de los diferentes componentes de la frontera recientemente indicados y mientras que la cristalización de la línea divisoria moderna encubría de cierta manera la constitución material de la propia frontera, su origen violento, nos encontramos actualmente con una situación en la que están expuestos a plena luz. Para decirlo en pocas palabras, tomar la frontera como un método, sobre la base de la presión concretamente ejercida sobre las fronteras por parte de migrantes y de refugiados en muchas partes del mundo, es un intento de lectura teórica y política de esta situación. Requiere una mirada que asuma la función constitutiva de las fronteras (su capacidad de enmarcar el mundo, su implicación en lo que llamamos en el libro fabrica mundi), pero al mismo tiempo capaz de centrarse en los conflictos, los choques, los conjuntos de relaciones que a menudo la frontera misma tiende 
a ocultar. El énfasis en la naturaleza relacional de las fronteras es particularmente importante en esta nueva mirada. Jugando con la definición del capital de Marx, a menudo decimos que la frontera no es una "cosa" sino un conjunto complejo de relaciones sociales mediadas por "cosas" (que incluyen muros, vallas, alambrados, tecnologías de control militarizadas y digitalizadas, pero también puentes y pasarelas). Es observando las mutaciones de estas relaciones sociales que la frontera se convierte en un punto de vista efectivo en torno a los conflictos más intensos que dan forma a la globalización contemporánea.

\section{Pese a la mencionada diversificación de las fronteras, el discurso político - pero también algunos estudios críticos- insiste en adoptar el muro como ícono pa- radigmático de las fronteras contemporáneas ¿A qué atribuirían esta obsesión con los muros, las vallas, los alambrados?}

Bien, en primer lugar, creemos que es importante destacar que en los últimos años hemos presenciado una impresionante multiplicación de muros. Basta con leer, por ejemplo, el libro de Wendy Brown, Estados amurallados, soberanía en declive ${ }^{4}$, para tener una idea del asunto. Este es un proceso al que tenemos que enfrentarnos, pero, de alguna manera, al igual que Wendy Brown, creemos que centrarse en el muro como una especie de paradigma de la realidad contemporánea de las fronteras puede ser engañoso. En su existencia concreta y, de cierta manera, majestuosa, el muro tiende a servir como icono de un poder que está fuera de ahí, en una dimensión trascendente para ponerlo filosóficamente. Es la mejor ejemplificación [instansiation] de una "cosa" a la que se puede reducir la frontera y, tal como mencionamos anteriormente, creemos que esta no es la mejor manera de analizar críticamente las fronteras. Además, el muro facilita una comprensión de la frontera centrada en su poder de exclusión. Es necesario, por supuesto, analizar la exclusión realizada y reforzada por las fronteras, pero estamos convencidos de que esto debe hacerse teniendo en cuenta simultáneamente las formas de inclusión diferencial y jerárquica que estas, a su vez, permiten, investigando en general las transformaciones de los mismos patrones de inclusión social que estas formas señalan. Este punto es muy importante para nosotros ya que es un buen ejemplo de lo que queremos decir con la "frontera como método". Incluso si tomamos los aspectos más intimidantes de los muros -digamos,

4 BROWN, W, Estados amurallados, soberanía en declive. Barcelona: Herder Editorial, 2015. 
el que circunscribe los territorios ocupados en Palestina o el que rodea vastos trechos de las fronteras entre México y los EE.UU.-, veremos que lo que no pocos análisis críticos enfatizan es la articulación, en tales casos, de procesos de fortificación y de exclusión con otros dispositivos y tecnologías de control que disciplinan y facilitan el cruce fronterizo. Enfocar esta articulación no relativiza ni disminuye la violencia encarnada en el muro, simplemente abre un ángulo más efectivo - creemos- desde el cual luchar contra él (¡con el objetivo de derribarlos!). Creemos que este es un punto importante particularmente en este momento, cuando uno piensa en el énfasis que Trump ha dado a la necesidad de completar el muro en la frontera con México. No debemos subestimar, por supuesto, las implicaciones concretas de tal énfasis, así como del anuncio continuo de las deportaciones masivas, pero también es necesario analizar tales implicaciones desde el ángulo proporcionado por dos nociones elaboradas por Nicholas De Genova, "espectáculo de la frontera" y "deportabilidad" . Los muros juegan papeles prominentes en el "espectáculo de la frontera", ellos contribuyen a reforzar la idea de una frontera completamente "cerrada", que pretende tranquilizar a parte de la población mientras que difunde el temor entre los migrantes potenciales (para quienes el cruce fronterizo se hace cada vez más peligroso) y los inmigrantes reales (que viven bajo el fantasma de la "deportabilidad"5, reforzado por el imaginario del muro). Pero insistimos: para entender estos roles, nuestra mirada no puede permanecer centrada únicamente en el muro físico o incluso en la frontera magnificada. Tiene que abarcar un sinnúmero de dispositivos fronterizos, cuyas operaciones pueden ser menos visibles, pero no por ello son, en definitiva, menos importantes que las del muro.

\section{¿Cuáles creen que son las implicaciones de la mencionada proliferación de fron- teras para nociones como soberanía, ciudadanía y Estado?}

La pregunta apunta a una de las principales apuestas de nuestro trabajo. Podemos comenzar diciendo que las tres nociones políticas modernas que usted menciona son todas nociones limitadas; ellas se sustentan en la existencia de las fronteras. Esta simple observación cuestiona la marginalidad de las fronteras en la teoría política, que de alguna manera corresponde a la ubicación en el mapa de las fronteras como el margen de los territorios. Es fácil demostrar que un territorio, en el sentido moderno

5 DE GENOVA, N, The deportation regime: Sovereignty, space, and the freedom of movement. En De Genova and Peutz, eds., 2010, 33-65. 
de una unidad limitada y homogénea (legal y políticamente), no podría existir sin el trazado de una frontera [border]. Y esto demuestra una vez más la naturaleza constitutiva y productiva de las fronteras. Pero quizás sea más importante enfatizar aquí que hoy en día nos enfrentamos a potentes procesos que desafían y distorsionan, de manera tendencial, precisamente la unidad y homogeneidad de los territorios nacionales. Esta es la razón por la cual estamos muy interesados en el estudio de la multiplicación de los enclaves, zonas económicas especiales formales e informales, corredores logísticos, que es posible observar en muchas partes del mundo (incluyendo en América Latina). Estamos convencidos de que tales procesos de "heterogeneización" de los territorios -cada uno de ellos basado en dispositivos fronterizos específicos-, son un componente fundamental de las transformaciones espaciales generadas por los procesos contemporáneos de la globalización capitalista. Dentro de estos procesos, los estados continúan desempeñando papeles importantes, lo cuales analizamos en un nuevo libro que acabamos de terminar, The Politics of Operations. Excavating Contemporary Capitalism ${ }^{6}$. Ellos pueden incluso reclamar un nuevo protagonismo -como es actualmente el caso, y ello ocurre desde los EE.UU. a la India, o desde Turquía a Rusia-, pero se ven obligados a aceptar de una u otra manera los procesos globales de valorización y acumulación de capital que corresponden a una arquitectura global de producción. Muchos de los espacios que acabamos de mencionar-desde las zonas económicas especiales hasta los corredores logísticos- están regulados de acuerdo con los ordenamientos jurídicos y las normas técnicas que fomentan la aparición de nuevas combinaciones de soberanía, gubernamentalidad y gobernanza. Esto es lo que ocurre también a lo largo de las fronteras en muchas partes del mundo, donde el poder del Estado definitivamente sigue reclamando su prerrogativa soberana sobre las fronteras, pero al mismo tiempo se involucra en "ensamblajes" híbridos y no necesariamente estables (para usar la palabra de Saskia Sassen) con otros actores -desde actores humanitarios hasta empresas privadas y organizaciones intergubernamentales como la OIM-. Investigar las mutaciones de la soberanía en la frontera (tomando la frontera como un "método") arroja luz sobre formas específicas y a menudo aterradoras de lo que Michael Hardt y Toni Negri describieron en el año 2000 como una "constitución mixta" del "Imperio". Hacemos hincapié en la continua relevancia de la soberanía, pero al mismo tiempo tratamos de investigar sus

6 NIELSON, B y MEZZADRA, S, The Politics of Operations. Excavating Contemporary Capitalism. Durham: Duke University Press, 2017.

7 HARDT, M y NEGRI, T, Imperio. Madrid: Paidós, 2002 [2000]. 
mutaciones, mutaciones que surgen de nuevos desarrollos políticos y jurídicos, pero también constituyen la presión de las operaciones globales del capital. Y tomamos la misma postura con respecto a la ciudadanía, un concepto que durante las dos últimas décadas ha estado en el centro de muchos estudios críticos. Para decirlo brevemente, consideramos la ciudadanía como un campo de tensiones, de lucha. Analizamos la alteración de la "ciudadanía social" asociada con el Estado de Bienestar o de desarrollo, e intentamos arrojar luz sobre la multiplicación de posiciones dentro del mismo espacio de ciudadanía -que corresponden al establecimiento de nuevas jerarquías y nuevas formas de inclusión diferencial-, para usar nuevamente nuestra noción. Al mismo tiempo, también observamos la ciudadanía desde el ángulo de las luchas y de los movimientos, considerando por ejemplo las prácticas y reivindicaciones de los "migrantes ilegalizados", así como también las luchas por el "derecho a la ciudad" y en contra de la expansión de los confines [frontiers] de la gentrificación. Aun así, somos muy críticos con respecto a la ciudadanía como forma predominante de subjetividad política, y estamos más interesados en seguir trabajando en el cruce entre los conceptos de clase y multitud en nuestra búsqueda de un sujeto de política radical para el presente.

\section{La proliferación de las fronteras va de la mano con la heterogeneización del espacio global y de la multiplicación de las formas de trabajo ¿Qué desafíos plantea esta gramática de lo múltiple para la práctica política?}

Como ya hemos discutido en algún grado de detalle los conceptos de proliferación de fronteras y de heterogeneización del espacio, tiene sentido responder a la pregunta discutiendo la importancia político práctica del concepto de multiplicación del traba$j o$. En primer lugar, debemos, sin embargo, señalar que la proliferación y la heterogeneización son conceptos distintos al de la multiplicación. Mientras que la proliferación describe un incremento en número, la heterogeneización implica un aumento en las diferencias de modo. La multiplicación los incluye a los dos, pero añade otro elemento. Es un concepto matemático que implica la posibilidad de división.

Es importante destacar que nuestro interés específico en la multiplicación del trabajo surge de un intento por repensar el concepto de división internacional del trabajo, que tiene una historia respetable en la economía política y fue elaborado críticamente por Marx en el contexto de su discusión sobre cómo el concepto de capital implica 
necesariamente un mercado mundial. A comienzos de los años ochenta, una serie de pensadores empezaron a hablar de una nueva división internacional del trabajo, en específico, como forma de describir analíticamente los cambios ocurridos en el mundo de la producción con la aparición de cadenas de suministro coordinadas logísticamente y el desplazamiento de muchas manufacturas industriales hacia partes del mundo donde el costo de la mano de obra era menor. El poner nuestra atención en las formas en que este escenario ha mutado en el mundo contemporáneo fue lo que nos llevó a forjar el concepto de la multiplicación del trabajo. Para nosotros, el concepto tiene tres cursos de desarrollo distintos pero relacionados. El primero describe la diversificación espacial del trabajo a través, más allá y entre las fronteras que proliferan, que ya no pueden identificarse exclusivamente con la línea territorial del Estado-nación. El segundo registra la intensificación del trabajo (labor) -o su extensión más allá de los límites temporales de la jornada laboral-, como fue descrito por Marx, y en el tiempo de la vida. Y la tercera pone de relieve la heterogeneización del trabajo o su expansión más allá de la norma legal del trabajo asalariado "libre" en formas nuevas y antiguas de precariedad y coerción.

En términos políticos prácticos, esta elaboración de conceptos bajo el signo de lo múltiple es solo un punto de partida, no creemos que el mero reconocimiento o celebración de la multiplicidad sea un acto político efectivo. Sin embargo, pensamos que es un paso necesario para una política efectiva, y esto significa reconocer la raza y el género al igual que la clase como formas de sujeción política y arenas en las cuales las luchas por la liberación deben tomar forma. El desafío que la multiplicidad presenta para la política dice relación con la fragmentación y la diferenciación. La fuerza política se suele entender como un efecto de unidad. Conocemos bien y aceptamos las razones intelectuales y políticas para alterar este recurso irreflexivo de unidad como una figura de posibilidad política, ya sea que implique la sedimentación de ontologías unívocas en mil mesetas, el desmantelamiento psicoanalítico del monoteísmo o el reconocimiento más directo de que el forjamiento de la unidad siempre implica dinámicas violentas de exclusión e inclusión. Pero no queremos ser demasiado insistentes en ignorar las lecciones históricas de la organización y de los logros políticos prácticos de las fuerzas sociales que luchan por la unidad y la coordinación dentro y a pesar de la discordia interna. 
Moverse entre la mayoría y la minoría es el desafío político, y tiene muchas resonancias prácticas, como puede verse fácilmente en algunas de las luchas más impresionantes del presente, incluyendo las nuevas movilizaciones feministas que son particularmente fuertes en América Latina. Creemos que los procesos de traducción son importantes para enfrentar este desafío, siempre y cuando se entiendan en un sentido extra-lingüístico y no resulten en prácticas sociales que generen comunidades claras y distintas trazando líneas entre ellas. Prácticamente esto significa que la política no debe temer los descarrilamientos de la comunicación o los encuentros con las fronteras que separan las luchas políticas entre sí. Estamos interesados en las luchas en las que el desarraigo [tear] estableció subjetividades políticas distanciadas de sí mismas, abriendo nuevas perspectivas de organización mediante el trabajo a través de y sobre las fronteras. No limitamos estas luchas al campo de la política migratoria, pero reconocemos el papel crucial de la migración en los intentos contemporáneos de repensar la forma organizativa de las luchas dentro y contra el capitalismo. Es en este sentido que hablamos de luchas fronterizas.

\section{El control de fronteras y la gestión de la migración tienen directa relación con} la creación de mercados laborales. Sin embargo, al ver de cerca las prácticas constituidas en los últimos años en torno a la migración, tales como centros de internamiento, programas de deportación, métodos cada vez más sofisticados de vigilancia y de control, técnicas de traslado (coyotaje), entre otras, ¿no podríamos hablar también de mercados o industrias migratorias?

Ciertamente estamos de acuerdo en que los controles fronterizos y la gestión de la migración son herramientas importantes para la producción de los mercados laborales. El hecho de que los mercados de trabajo sean concebidos principalmente en términos de delimitaciones nacionales es un efecto directo de dicho control y gestión, y es importante rastrear las dimensiones históricas y la evolución de esta situación, así como sus efectos contemporáneos.

Las historias de esclavitud, de servidumbre [indenture] y de otras formas de trabajo "forzado" [bridled] son cruciales a este respecto no solo porque desplazan la figura del trabajador asalariado "libre" del centro de la lucha histórica, sino también porque proporcionan importantes puntos de contraste para cualquier confrontación con la migración forzada en el mundo contemporáneo. Es significativo, por ejemplo, que 
tendamos a concebir el mercado financiero en términos globales, mientras a los mercados de trabajo los reducimos a una escala de nivel nacional. Así como es importante registrar la interrelación de las economías nacionales en el mundo contemporáneo - por ejemplo, el engranaje de las economías estadounidense y china en términos de finanzas y tecnología- sería miope también pretender que el cálculo agregado de las fuerzas económicas a escala nacional no produce efectos reales. Las cuantificaciones abstractas tales como el PIB, la tasa de empleo o el salario mínimo asumen una economía articulada, pero esta delimitación no es meramente un supuesto conveniente, tiene efectos y consecuencias absolutamente materiales, y la frontera es la cristalización social e institucional de esta materialidad. La frontera crea y produce la economía nacional y, en este sentido, las naciones consideran el control de las fronteras y de la migración no solo como una prerrogativa soberana del Estado, sino también como una técnica (a menudo moralizada) de calibración económica. La fantasía del equilibrio del mercado requiere y asume la violencia de la frontera -y esto es particularmente evidente en el caso de los mercados laborales-. Sin embargo, esta fantasía siempre se ve perturbada por la misma naturaleza y producción de la mercancía que se intercambia en los mercados de trabajo, es decir, la fuerza de trabajo. Debido a que esta mercancía es inseparable del cuerpo de su "portador", crea todo tipo de factores de intromisión -incluyendo los efectos sociales y culturales- que alteran la visión de la frontera como una válvula de funcionamiento perfecto o dispositivo de filtración que permite la configuración técnica del mercado de trabajo. Lo que significa que los movimientos migratorios y la fuerza de trabajo que movilizan llegan a ser percibidos como un exceso turbulento que siempre necesita mantenerse a raya. Las tecnologías políticas que menciona -detención, deportación, formas cada vez más sofisticadas de monitoreo y programas de traslado- están todas encaminadas a contener este exceso. Creemos que es realmente útil analizar estas técnicas como parte de un mercado o industria migratoria, aunque es importante subrayar que son aún técnicas políticas y que su forma institucional actual en el mundo que asistimos toma cada vez más la forma de una asociación público-privada. En algunos casos, lo que se intercambia en estos mercados no es solo dinero, sino también formas de influencia y poder político - pensemos, por ejemplo, en los "intercambios de refugiados" ["refugee swaps"] que se producen entre la Unión Europea (o más concretamente Alemania) y Turquía o Australia y los EE.UU-. 
Más en general, la industrialización y la mercantilización de los movimientos migratorios es un fenómeno que hemos intentado analizar con camaradas y colegas como Manuela Bojadzijev y Ned Rossiter, al investigar las relaciones entre migración y logística. Una vez que la migración es relacionada con las técnicas y las tecnologías logísticas que facilitan el movimiento de personas y de bienes alrededor del mundo, obtenemos algunos ángulos analíticos nuevos desde los cuales entender y actuar dentro de la política migratoria. Por ejemplo, en términos logísticos, el negocio de traslado de personas a través de acuerdos sobre la "trata de personas" y deportación toma dimensiones muy similares, pese a las muy diferentes relaciones con el poder estatal soberano que usualmente se supone que tienen. Del mismo modo, si entendemos la detención como un acuerdo industrial que involucra cadenas de suministro de diferentes actores públicos y privados, ganamos nuevas formas de acción políticas para enfrentarlos, por ejemplo, conduciendo boicots contra entidades que se sitúan en la cadena de suministro de acuerdo con acciones políticas necesarias y acciones simbólicas conocidas, tales como escalar o derribar las vallas que rodean los centros de detención. Siempre es necesario entender, sin embargo, que los mercados no se equilibran solos. Tales acciones no pretenden tanto alterar un sistema de manera blanda [smooth] cuanto exponer sus desequilibrios inherentes e identificar vetas y espacios de fricción donde la logística de las industrias migratorias puede ser confrontada y combatida.

Cuando se trata de la migración y del refugio los relatos e incluso los análisis académicos suelen centrarse en la victimización (guerra, pobreza, desastres naturales, mafias, trata, explotación, esclavitud), pero olvidan que también se producen relatos y prácticas de subjetivación política ¿Qué implica la frontera como fabrica mundi?

Siempre nos hemos resistido a la tendencia que busca presentar a los migrantes como víctimas, aun cuando reconozcamos que muchas demandas estratégicas y causas nobles puedan sostenerse [served] sobre una perspectiva como esta. Al igual que muchos otros pensadores, nos hemos negado a utilizar los términos acuñados por los Estados y las organizaciones internacionales para clasificar diferentes tipos de migrantes -refugiados, migrantes económicos, migrantes cualificados, etc.-, excepto en los casos en que deseamos específicamente discutir los efectos creados por estas categorías. Para nosotros, trabajar a lo largo de todo el espectro de la migración es 
una necesidad analítica. No creemos que sea posible comprender a cabalidad la difícil situación de aquellos que huyen de los desastres naturales o que son víctimas de tráfico sin tener en cuenta las políticas de movilidad vinculadas con las formas de migración de alto nivel, en particular aquellas asociadas con la vida transnacional de las élites empleadas por corporaciones multinacionales. Esta es una de las razones por las que en La frontera como método realizamos dos tipos de análisis de la migración, y que a menudo se sitúan en extremos opuestos del espectro -los traders financieros y las trabajadoras domésticas y de cuidados. Nuestro objetivo en la realización de este análisis fue revelar las relaciones y resonancias entre estos dos tipos muy diferentes de migración. Sin embargo, es importante señalar que las posiciones en este espectro no pueden correlacionarse fácilmente con las tensiones de sujeción y subjetivación que surgen con los movimientos migratorios. Efectivamente pensamos que los propios movimientos migratorios tienen efectos políticos importantes, sean o no intencionados por parte de los sujetos involucrados (por cierto, esta es una hipótesis de investigación que ha sido probada con respecto a América Latina en un libro colectivo de pronta publicación, libro que uno de nosotros ha coeditado junto a dos camaradas y colegas mexicanos ${ }^{8}$ ). Al mismo tiempo, hacemos hincapié en la necesidad de un enfoque que trabaje a través y desde las posiciones subjetivas de los migrantes, siempre y cuando el análisis siga atento simultáneamente a las condiciones estructurales que afectan la movilidad. Una parte importante de este esfuerzo es llevar a cabo un análisis que documente y honre las luchas organizadas llevadas a cabo por los migrantes - y esto es algo que hemos hecho investigando luchas como las de los sans papiers en Francia durante los años noventa-. También somos sensibles a la necesidad de dar cuenta de los modos de subjetivación expresados a través de luchas fronterizas más espontáneas, como las revueltas [riots] entre las comunidades migratorias de segunda generación en Londres y París o las dirigidas por comunidades afroamericanas segregadas y desfavorecidas por las fronteras internas de Estados Unidos en ciudades como Ferguson. En tales casos, tenemos el cuidado de enfatizar la vasta e innumerable acumulación de experiencias y actividades políticas subterráneas que están detrás y producen la ruptura de un evento político que deriva en titulares, y que a la vez abre [shifts] posibilidades. Además, como ya hemos señalado, entendemos los movimientos migratorios en sí mismos como fuerzas políticas

8 CORDERO, B, MEZZADRA, S y VARELA, A, eds, América Latina en Movimiento. Autonomía de la migración, fronteras y nuevas geografías de lucha. Buenos Aires: Tinta Limón; México: UACM; Madrid: Traficantes de Sueños, 2017. 
que presionan y desafían las arquitecturas políticas y espaciales del territorio y la demarcación, que son a la vez la precondición y el producto de las formas contemporáneas del capitalismo global. Este punto de vista les parecerá a algunos romántico y celebratorio, pero siempre hemos enfatizado que este tipo de fuerza política no puede necesariamente ser descubierto en las experiencias y actitudes individuales de los migrantes, haciendo uso, por ejemplo, de métodos de investigación como la entrevista etnográfica. Los migrantes no ven necesariamente su movilidad como algo que excede a las operaciones del capital. Aquí nos encontramos ante la diferencia entre la subjetivación y lo que solía llamarse en la teoría marxista la conciencia [consciousness]. En nuestro análisis, la capacidad productiva de las fronteras, que tratamos de inscribir en la frase latina fabrica mundi, tiene dos dimensiones inseparables: la producción del espacio y la producción de la subjetividad. Así, entendemos la dinámica de sujeción y subjetivación inherente a los movimientos migratorios, tan profundamente implicada en una serie de problemas relacionados que van desde el funcionamiento de la globalización capitalista, los cambios en la estructura del "mercado mundial" y la división internacional del trabajo hasta los desplazamientos [shifting] de los roles y formas del Estado, la aparición de nuevas geografías de desarrollo y producción, las mutaciones en la expresión del poder político y los cambios en los contornos de la ciudadanía, la clase y la pertenencia política. Al trazar las relaciones entre, y la implicación mutua de, la producción del espacio y la producción de la subjetividad, buscamos métodos para reformular la política de las fronteras y la migración mucho más allá de los "mundos limitados" de las teorías de la justicia que se constituyen en una comunidad política como un Derecho fundamental sobre el cual todo otro derecho se construye.

\section{¿Cómo la migración y las fronteras pueden contribuir al debate sobre las polí- ticas de lo común?}

Ya hemos discutido en qué medida nuestro interés en la multiplicación del trabajo está relacionado con la política de la división. Es importante destacar que la división es una acción frecuentemente atribuida a las fronteras. Las fronteras, oímos con frecuencia, dividen un "nosotros" y un "ellos", un adentro y un afuera, un territorio de otro, y así sucesivamente. Pero las fronteras no solo dividen, también conectan, existen en la cúspide de la división y de la conexión, y en consecuencia también plantean la cuestión de lo que significa compartir o "repartir algo". La división, y con ello la 
multiplicación, plantea así la pregunta por el cómo vivimos juntos, cómo dividimos el espacio, el tiempo, los recursos y la socialidad [sociality], eso que hoy se describe a menudo como la producción de lo común. Nuestra propia intervención dentro de los debates en torno a este tema en particular se centran, por un lado, en este aspecto de la producción (ya que estamos convencidos de que incluso los "bienes comunes" más materiales como la tierra y el agua tienen que ser producidos políticamente para existir como bienes comunes, lo que significa, por ejemplo, que hay que inventar una regla pragmática para su gestión y para acceder a ellos), y, por otro, en el tipo de subjetividad que se necesita para impulsar una política de lo común. En este sentido, la cuestión de la diferencia y de las fronteras es nuevamente fundamental y tomamos muy en serio la lección de las feministas postcoloniales y negras como Chandra Talpade Mohanty y Audre Lorde, quienes subrayan la importancia de estas cuestiones para la teoría y la práctica política e insisten en que reivindicar las diferencias como puentes solo puede hacerse luchando simultáneamente contra ellas en tanto lo que son, barreras, fronteras que nos dividen. Esto implica para nosotros una suerte de politización de la noción y la práctica de la traducción, como una herramienta capaz de sostener la reivindicación de las diferencias y la producción de lo común. Pensamos, una vez más, que tal lección resuena y es un desarrollo creativamente más profundo dentro de movilizaciones feministas recientes en varias partes del mundo. Al mismo tiempo, si hemos de tomar en serio el desafío de una política de lo común, es evidente que tenemos que repensar y relanzar una crítica radical de la propiedad privada. En La frontera como método enfatizamos en la importancia constitutiva de las fronteras -tanto histórica como conceptualmente-, para el establecimiento y el funcionamiento de la propiedad privada. Las luchas fronterizas, tal como las pensamos, nos ayudan a reenmarcar la crítica de la propiedad privada, lo que significa, al fin y al cabo, una reinvención del comunismo. 